\title{
A simulation-based design paradigm for complex cast components
}

Received: 23 December 2005/ Accepted: 2 May 2006/Published online: 24 August 2006

(C) Springer-Verlag London Limited 2006

\begin{abstract}
This paper describes and exercises a new design paradigm for cast components. The methodology integrates foundry process simulation, non-destructive evaluation (NDE), stress analysis and damage tolerance simulations into the design process. Foundry process simulation is used to predict an array of porosity-related anomalies. The probability of detection of these anomalies is investigated with a radiographic inspection simulation tool (XRSIM). The likelihood that the predicted array of anomalies will lead to a failure is determined by a fatigue crack growth simulation based on the extended finite element method and therefore does not require meshing nor remeshing as the cracks grow. With this approach, the casting modeling provides initial anomaly information, the stress analysis provides a value for the critical size of an anomaly and the NDE assessment provides a detectability measure. The combination of these tools allows for accept/reject criteria to be determined at the early design stage and enables damage
\end{abstract}

\section{S. P. A. Bordas}

Ecole Polytechnique Fédérale de Lausanne (EPFL), Institut des Structures, Laboratoire de Mécanique des Structures et des Milieux Continus (LSC), Station 18, 1015 Lausanne, Switzerland

S. P. A. Bordas $(\square)$

Civil Engineering Department, University of Glasgow, Glasgow G12 8LT, UK

E-mail: stephane.bordas@alumni.northwestern.edu

Fax: + 44-141-3304557

J. G. Conley

Northwestern University, Kellogg School of Management, 5249 Jacobs Center, Evanston, IL 60208-3111, USA

B. Moran

Mechanical Engineering Department, Northwestern University, Evanston, IL 60208, USA

J. Gray

Iowa State University, Center for NDE, Ames, IA, USA

E. Nichols

Vought Aircraft Industries, Dallas, TX, USA tolerant design philosophies. The methodology is applied to the design of a cast monolithic door used on the Boeing 757 aircraft.

Keywords Casting design and modeling Extended finite element method, XFEM - Crack growth and damage tolerance analysis $\cdot$ Non-destructive evaluation - Industrial problems · Micro-macro simulations

\section{Introduction: cast airframes components and the regulatory design regime}

As an example of the proposed design paradigm, we will discuss an aerospace industry example, where design specifications and performance are, in the USA, subject to the review and approval of the corresponding regulatory authorities (FAA, JAA, etc.). Airworthiness standards, in the United States, are governed by Title 14 of the US Code of Federal Regulations (14 CFR). Chapters I and II of this publication are typically referred to as the Federal Aviation Regulations (FARs). In particular, the 14 FAR Parts 25 and 33 are airworthiness standards for transport category airplanes and aircraft engines, respectively. The FAR 25 mandates that all primary structure airframe component design must satisfy the principles of damage tolerance (14 CFR 25.571).

As per these regulations, airframe components made by foundry processes (castings) must meet the same strength requirements as other metallic structures, wrought or assembled, such as material characterization, static strength, durability and damage tolerance. Issues that contribute to the regulatory satisfaction of these requirements include foundry production, control and inspection methods. The variability of cast alloy material properties within a casting and across castings of the same configuration has hindered the establishment of statistically valid allowables [1]. To account for the variability, an additional, inherently redundant, 
factor of safety is a design requirement when castings are used in certain applications. This safety factor is henceforth referred to as the casting factor $(\mathrm{CF})$. In certain, very controlled instances, it is possible, with agreement of the FAA to reduce the CF to 1.0. Several critical structural castings were designed with a $\mathrm{CF}$ of less than 1.5 or unity [2-5]. CFs are imposed on aerospace casting designs to achieve an increased margin of safety that counts for historic uncertainties in the strength of cast materials. The net effect of the CF is an increase in cast component volume and weight and a loss of competitiveness of casting technologies. The improvement of casting technology over time has encouraged the use of castings without a $\mathrm{CF}$ to make castings competitive and more widely used in fatiguecritical aircraft structures [2-5].

While castings have long been viewed as poor quality components, in recent years, casting processes have much improved, leading to a re-evaluation of their use. Indeed, the cost savings realized in using quality cast parts are substantial $[2,4]$.

An initial concept for an integrated simulation based design system for safety critical castings such as automotive parts was first presented by Conley et al. [6]. According to the methodology, casting process knowledgeable designers work in concert with airframe designers (mechanical performance and crack growth expertise) and non-destructive evaluation professionals so that respective design decision content can be efficiently exchanged and integrated. This approach shows promise for evaluating the damage tolerance and safety of critical airframe components. Briefly, the approach uses a combination of foundry process simulations to predict cast anomalies, crack growth modeling methods (extended finite elements) to predict the effect of the casting anomalies on fatigue life and residual strength, and modeling of non-destructive evaluation (NDE) processes to determine the inspectability of both initial anomalies and potential cracks that may grow in-service. A graphical representation of the integrated design framework is given in Fig. 1. The method begins with the component's computer aided design (CAD) solid model generated in a commercial software package. The solid model is imported to a casting simulation package, static and dynamic structure analysis, and NDE simulation codes at various times throughout the development cycle to examine questions such as the size and location of anomalies, the effect on mechanical properties, probability of detection, etc. Appropriate integration and application of the methods establishes a more informed basis for in-plant accept/reject criteria. The possibilities of this system are promising and raise the following important research questions.

- What computing power challenges and opportunities exist for this type of design system?

- Given a particular airframe component geometry and foundry process parameters, where are the casting microstructure anomalies likely to occur?
- Is it possible to couple both macro and microprocess simulation to enable the prediction of both the size and location of the anomalies?

- How fast do cracks grow from these anomalies and are any of them critical, i.e., compromising the residual strength of the part under in-service loading conditions?

- Is it possible to simulate how anomalies potentially impact the damage tolerance and or inspectability of the part?

- Can we simulate and predict the detectability of such anomalies using NDE methods?

- With all of the above, can we now begin to address the effects of the cast anomalies and hence quantify the mechanical behavior of cast airframe component designs in a manner that brings scientific reasoning to the aforementioned uncertainty about suitable accept/ reject criteria?

In this paper, the integrated approach is described through its application to the geometry of the Boeing 757 electrical equipment (EE) access door, a monolithic casting with integral skin poured from D357 aluminum alloy treated to the T6 condition (solution annealed and aged to peak mechanical properties). With information available to the designer at the early stages of product or concept design, an improved understanding of casting design tradeoffs and the optimal design may be quantitatively recognized and assessed.

\section{Component geometry and design system}

The central definition of component geometry in most commercial computer automated design systems is the

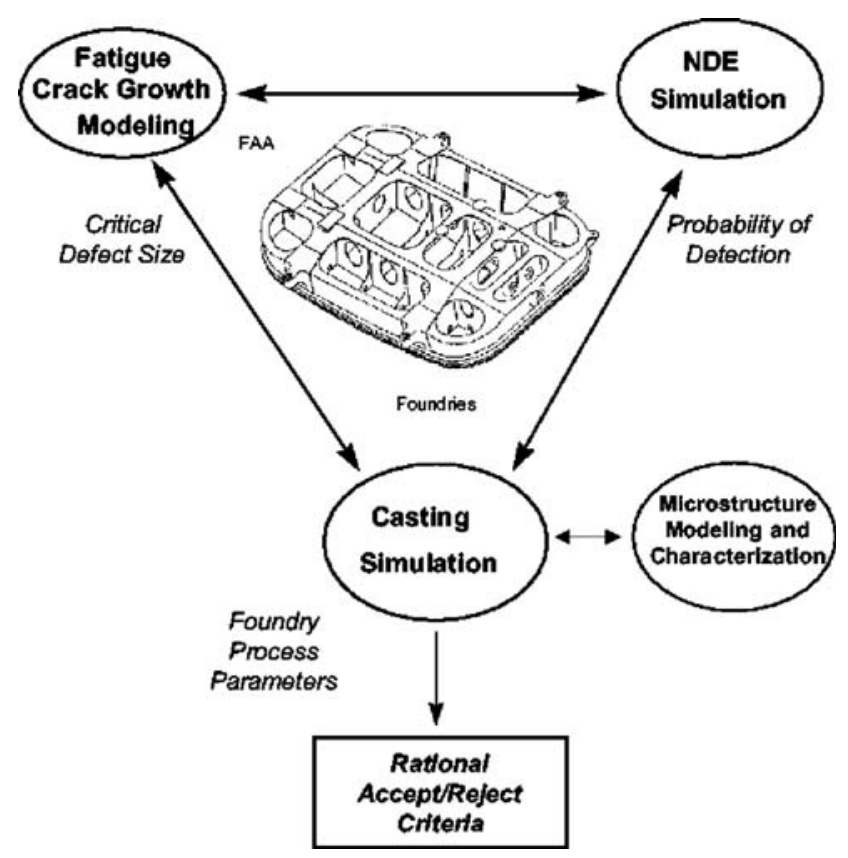

Fig. 1 Novel design paradigm, integrating NDE simulation, casting modeling, and damage tolerance simulation 
CAD solid model. The B757 EE Access door is a monolithic casting with an integral skin, many thin ribs and complex cross-sections as shown in Fig. 2.

The door itself provides access to the underside of the cockpit control panel for service and maintenance. Since this door is located on the underside of the B757 fuselage, design criteria must satisfy "ditch" loads that may be experienced in the event of a water landing. Such loads are transferred across a gasket sealing area and four regularly spaced stops on the perimeter of the door geometry. Therefore, the stops become areas for safety critical performance and reliability analysis.

\section{Casting modeling}

3.1 Filling and solidification process simulations of the Boeing $757 \mathrm{EE}$ access door

The material used to produce the door is the Aluminum alloy D357. Because of the existence of very thin walls $(2.2 \mathrm{~mm})$ within the casting, special attention is required when building control volume finite difference mesh for the Boeing $757 \mathrm{EE}$ Access door casting. The model includes the running system and the risers, all integral elements of the prefinished casting. The running system is the channel in which the liquid alloy flows to the mold cavity and determines the filling and solidification patterns within the overall casting. The riser on the top of

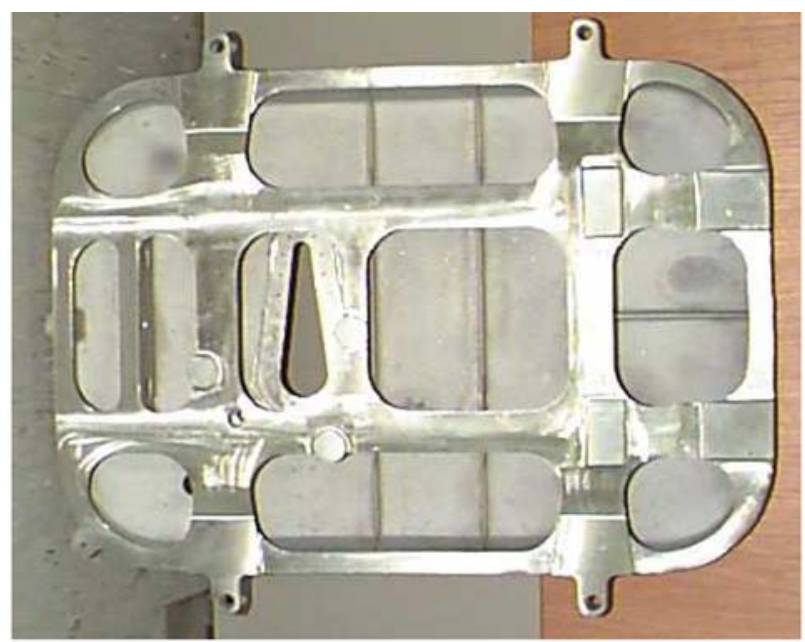

(a) Picture of door

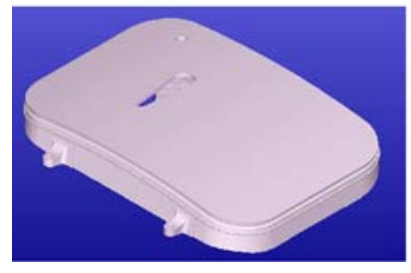

(b) CAD model: top view

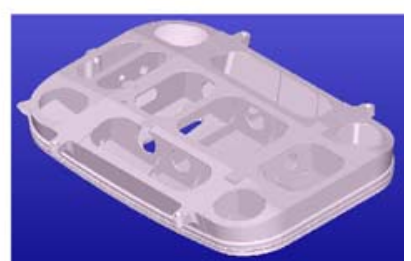

(c) CAD model :bottom view
Fig. 2 Geometry of the Boeing 757 EE Access door the casting is used to compensate for metal shrinkage and serve as the channel for gas ejection from the mold.

The total number of cells in the mesh, shown in Fig. 3 is $135,000,000$, the largest model generated to date with the commercial package. The size of the mesh alone presents considerable challenges from the point of view of processing and post-processing. The amount of memory [random access memory (RAM)] required for the solidification analysis are such that parallel computing was required.

From a parallel computing point of view, the bottleneck of the analysis of the Boeing 757 EE Access door casting lies in the amount of RAM available on the control node of the computing network. This means that the maximum problem size that the parallel computer can tackle is limited by this amount of memory. In order to keep the model computationally tractable given the available hardware, the mesh density of the model had

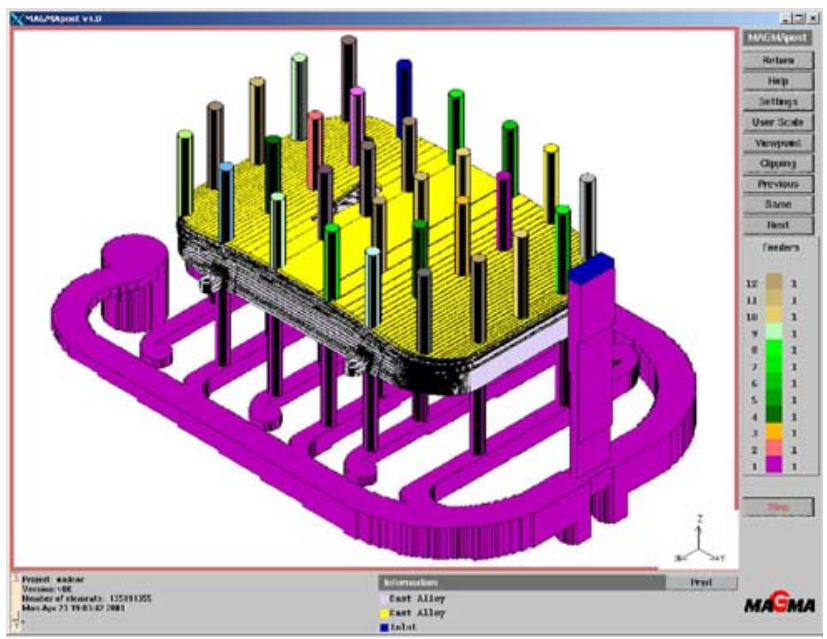

(a) Complete model

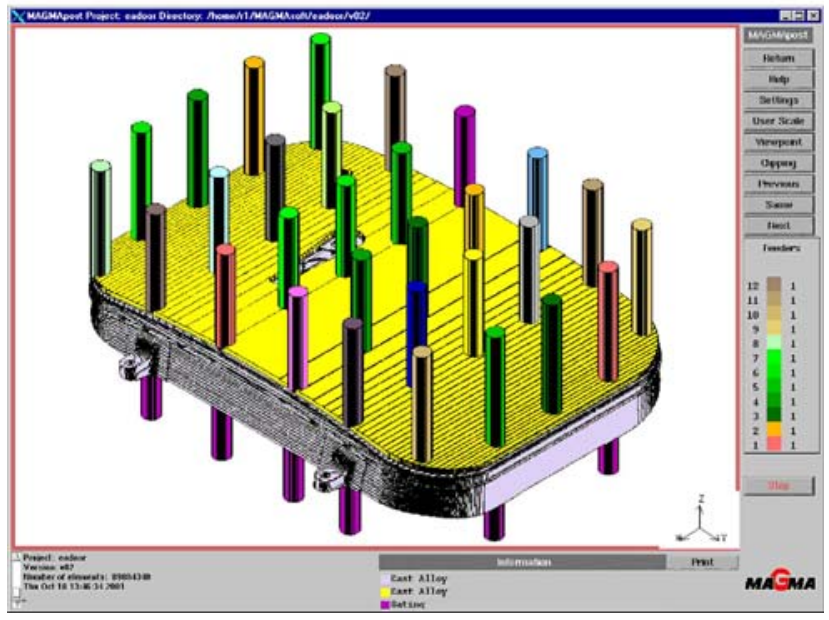

(b) Simplied model for foundry analysis

Fig. 3 Model and simplified model of the door in MAGMAsoft, for the purpose of this analysis, the runners, gating and risering system for this sand casting are generalized to avoid disclosure of proprietary foundry methods 
to be kept within acceptable limits given the hardware limitations. The mesh, modified from Fig. 3 for the purposes of simplicity totals $89,884,340$ cells. Elements of the downsprue and runner system in the original design were eliminated from the model and the inlet set at the bottom of each runner element under the door. With an equivalent static pressure on each of the inlets, the liquid Aluminum can enter the model from the bottom. The pressure equals the total weight of liquid aluminum in the casting, so that the molten metal exactly reaches the top of the risers.

The simulation results presented in what follows were carried out on an HP workstation with two processors and $10 \mathrm{~Gb}$ memory.

\subsection{Filling process simulation results}

Figure 4 shows the simulation results. Note that the analysis inputs and feeding/gating geometries used in the above analysis are not representative of the exact foundry methods used in the production of this component.
As such, the results presented below do not reflect on the quality of the production component. The analysis is presented merely as an example of what can be achieved with contemporary foundry simulation tools.

In the simulation illustrated in Fig. 4, the filling time is set to $20 \mathrm{~s}$. From the simulation results, it can be seen that the liquid metal entered the mold from the bottom, and then fills in the lower part as well as the side of the mold. Then, the liquid flowed to the middle of the part, and formed the flow field interface in the middle of the top of the door. These lines were formed near the solidus temperature, which could negatively impact the mechanical properties of the solidified aluminum in those areas.

Feeding efficiency is calculated based on the percentage filled of each control volume. From the translucent view of feeding efficiency shown in Fig. 5, several inefficiently fed areas with less than $100 \%$ feeding efficiency can be located in the casting.

The filling simulation is adequate in revealing such filling pattern deficiencies. This simulation result gives expression to the areas where feeding-related anomalies
Fig. 4 Results of the filling process simulation

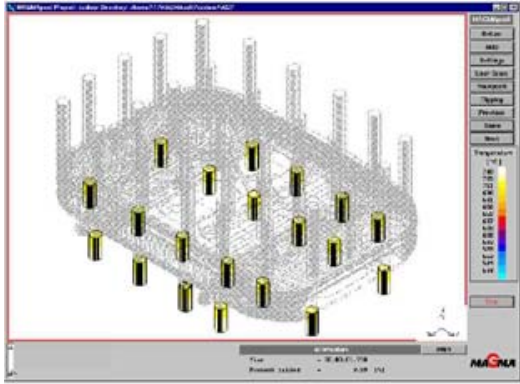

(a) $10 \%$ filled

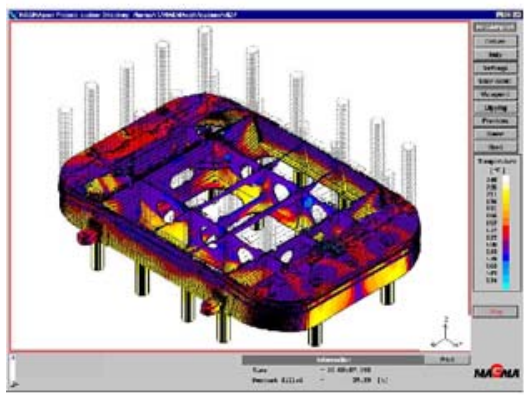

(c) $40 \%$ filled

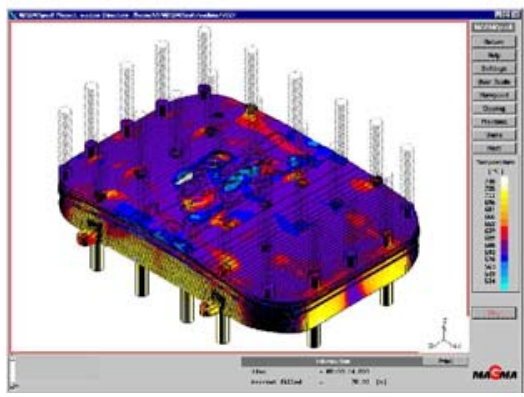

(e) $70 \%$ filled

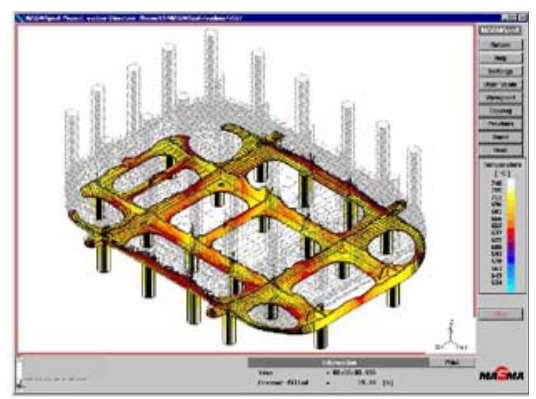

(b) $20 \%$ filled

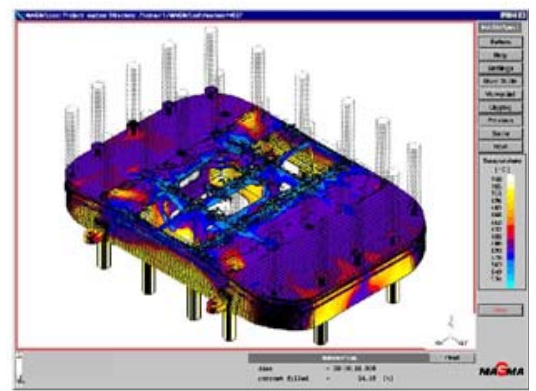

(d) $55 \%$ filled

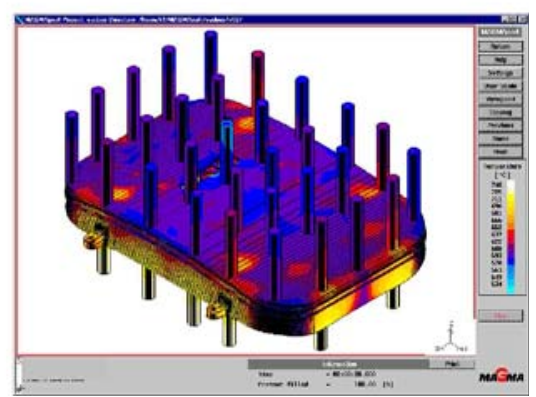

(f) $100 \%$ filled 
may possibly occur. Rigging adjustments to the model and subsequent simulations facilitates the virtual testing of many possible adjustments outcomes. Also, the flow field interfaces should be kept away from the casting areas with high stresses or low NDE inspection detectability.

3.3 Solidification process simulation results and exercise of the proposed design paradigm

Figure 6 is an example output from the solidification analysis. The simulation suggests the presence of cavities in the area of the door stops, which were shown, by the stress analysis, to be the location of the peak stresses in the structure (they withstand the reaction forces required to equilibrate the pressure on the door). Such cavities may reduce the strength of the component in those areas, and preclude its safe operation, especially if they grew into cracks and if their probability of detection were low. This is a good situation where the integration design method can be exercised.

In the context of the design paradigm of [6], the simulation results presented above serve as a basis for the damage tolerance assessment (DTA), providing approximate locations of potential pores or other anomalies. In turn, the NDE simulation can assess NDE coverage inspection requirements [7] of those initial anomalies and of the fatigue cracks that may grow, inservice, from those initial anomalies. Assume that the DTA simulation showed the anomalies predicted by the casting simulation model, decreases the residual strength of the component below a tolerable value or causes the formation of cracks that would become unstable be-

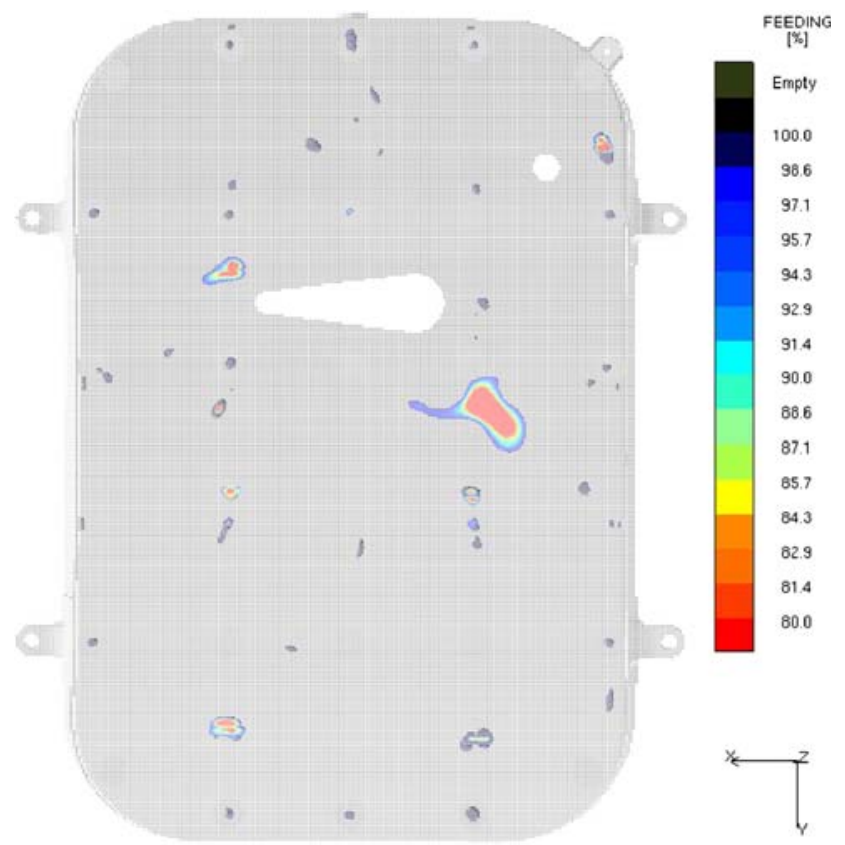

Fig. 5 Feeding efficiency (translucent, top view) tween two inspection events. It would then be possible to review the rigging and casting designs and/or the filling method to avoid the formation of such casting anomalies. Another alternative would be to make the nondestructive inspections of the part more frequent. The reliability of those inspections would be determined by the probability of a detection map yielded by the Non Destructive Evaluation simulations [7]. Another scenario would arise if the predicted anomalies did not endanger the safe operation of the component. In this case, the design of the casting may be improved by reducing the amount of material used in regions where the component was over designed. In concert with the foundries responsible for manufacturing the casting, wall thickness may be decreased, or bulk areas made more slender. On the whole, the proposed design paradigm [6] allows, after a few iterations, the overall improvement of the design of the component and a "converged", optimal solution to be achieved, that satisfies the static and fatigue strength requirements.

\subsection{Improvements in micromodeling}

Macromodeling, as described above gives the designer information on the location of anomalies. Micromodeling uses the macromodeling results to predict the shape, size and orientation of the anomalies. This is important since the geometrical characters of the anomalies will influence the ability to detect them, and determine the initial crack size used in the fracture mechanics simulations. Grains grow dendritically into shapes that are usually non-uniform and concave. Micromodeling is the first step towards an assessment of the complex geometry of anomalies.

The overall objective of micromodel development is to extend the time series thermodynamic macroscopic modeling output of filling and solidification simulations down to the scale of individual grain growth. Micromodels are hence coupled to macromodels with the

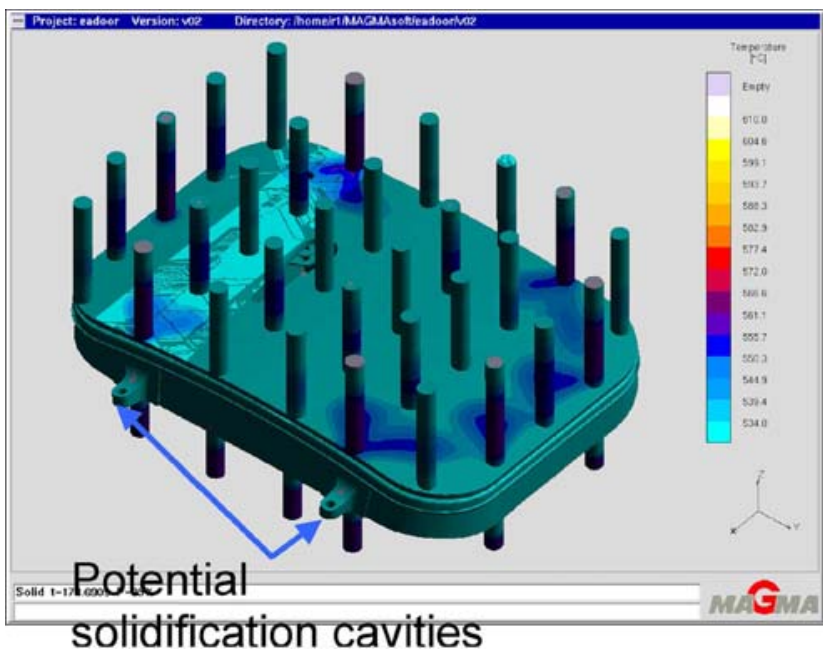

Fig. 6 Solidification process simulation results (95\% solidified) 
boundary conditions from the end of a macrosimulation becoming the initial conditions for a microsimulation.

Generally speaking, microsimulations seek to use the first principles of physics to model how the alloy solidifies at the level of individual grains. Since shrinkage cavities and other anomalies tend to form at the interfaces of such grains, the anomaly evolution follows from the grain evolution. While the macromodel (MAGMA soft simulation shown above) tells us where the anomalies are likely to be, the objective of micromodeling is to predict the size and orientation of anomalies in the area of concern, in this case the door stop.

As reported elsewhere [8-10], the micromodel development research employs an instantaneous nucleation model, which is complemented by the development of a similar, robust, two-dimensional (2D) micromodeling approach based on continuous nucleation authored by scientists at the University of Alabama Metals Casting Technology Center [11].

The micromodeling approach begins by taking the thermal conditions and history of a particular control volume located in a particular area of the casting and further meshing this geometry down to 2D cell sizes on the order of a few microns. On this scale, the dynamics of solidification and growth of grains from the melt in the presence of rapidly exsoluting gas is simulated. Recent effort to improve this approach to modeling was made, and a two and one-half dimensional model for grain shape, pore shape and pore size has been examined [11].

The two and one-half dimensional model for grain shape, pore shape and pore size has been setup based on the following assumptions:

1. Grains grow uniformly in all directions until they impinge upon some other grains.

2. The growth rate, $\dot{L}$ is the same for each grain.

3 . The numbers of nuclei, $n_{i}$, are the same in each plane.

4. Nucleation is instantaneous.

Because of assumption 3, nucleation occurs uniformly in the $z$-direction, though nucleation sites are randomly selected in the $x-y$ planes - shown in Fig. 7, the $z$ axis being normal to the planes represented in the figure. Grains nucleating on the $i$ th plane reach the 0 th plane at time $t$ after the nucleation, where

$t=i \frac{\mathrm{d} z}{\dot{L}}$.

The number of grains reaching the 0th plane during solidification is expressed as follows:

$N_{0}=n_{0}+2 \sum_{j=1}^{T \dot{L} / \mathrm{d} z} n_{j}$

where $T$ is the local solidification time, and $n_{j}$ is the number of nuclei on the $j$ th plane. As the number of nuclei is assumed to the same in each plane, $N_{0}$ can be calculated as follows:
$N_{0}=\left(1+2 \frac{T \dot{L}}{\mathrm{~d} z}\right) n_{0}$.

The product of the grain density (number of grains in unit area), $N$, and representative area, $A$, should be equal to $N_{0}$, and the equivalent diameter of a grain nucleated on the 0th plane should be $2 T \dot{L}$ at the end of solidification. Therefore, $n_{0}$ can be expressed in terms of $N$ and the maximum equivalent diameter, $D$ :

$n_{0}=\frac{N A \mathrm{~d} z}{d z+D}$.

In this equation, $N$ and $D$ can be determined experimentally.

A series of schematic diagrams showing growing grains in multiple planes are given in Fig. 7. Nucleation occurs instantaneously on each plane at the beginning of the process (time $=0)$. Three planes $(i=-1,0,1)$ are shown in the figure, and there are two nuclei on each plane $\left(n_{0}=2\right)$. Since grains grow uniformly in all directions at the same growth rate and the distance between each plane is equal to cell size in this example $(\mathrm{d} z=\mathrm{d} x=\mathrm{d} y)$, grains grow into neighboring planes when they grow one cell in an $x-y$ plane. Other planes do not have to be divided into cells for a resulting structure of one plane. In the 2.5-dimensional (2.5D) analyses, only the 0th plane is divided into cells.

Using this basic framework, 2.5D simulations were undertaken for the A356 casting alloy. Macrostructure

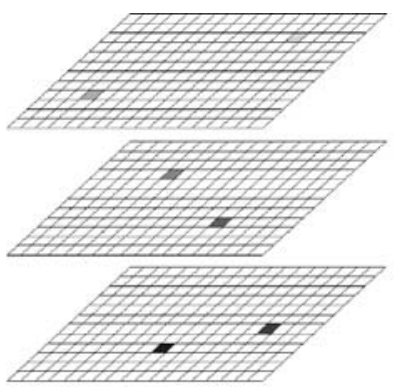

(a) Time $=0$

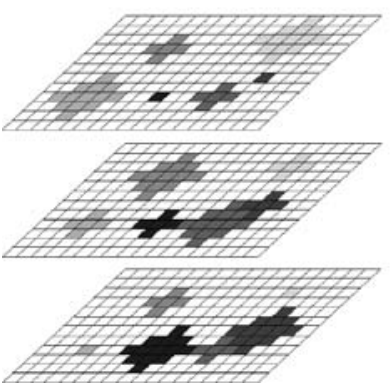

(c) Time $=2$

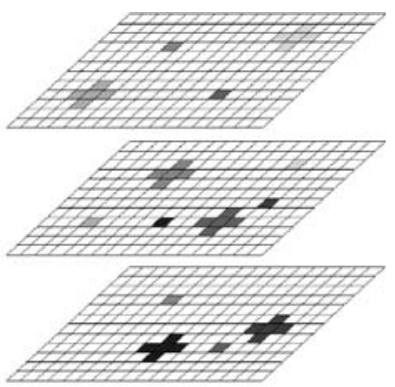

(b) Time $=1$

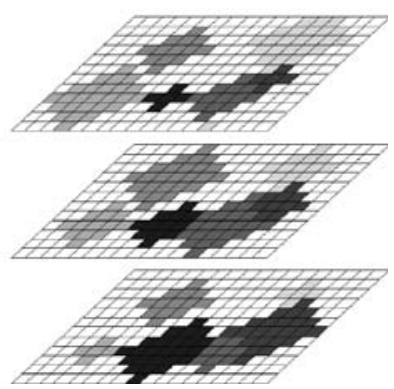

(d) Time $=4$
Fig. 7 Schematic diagrams showing concepts of grain growth process in multiple planes divided into cells. In the two and onehalf dimensional analysis, only the plane $(i=0)$ is divided into cells, but some grains on the plane are from other layers $(i= \pm 1$, 2, 3) 
predicted by the $2.5 \mathrm{D}$ simulation is compared with optical micrographs in Fig. 8. In the micrograph, Fig. $8 \mathrm{a}$, and the $2.5 \mathrm{D}$ result, in Fig. $8 \mathrm{~b}$, some small grains (under $200 \mu \mathrm{m}$ in diameter) are observed. Figure 8 shows that the $2.5 \mathrm{D}$ result is in agreement with the experiment.

The changes in the microsimulation model to achieve the $2.5 \mathrm{D}$ effects described above result in the following improvements:

1. Predicted porosity fraction is less scattered.

2. Two crystallographic orientations are considered in macroscopic grain structure evolution.

3. Temperature is determined by solid fraction.

4. Nucleation of gas porosity is not limited.

5. Gas porosity grows until gas pressures in each time step are balanced.

6. A two and one-half dimensional model provides more accurate grain size distribution than provided by the $2 \mathrm{D}$ model.

\section{Static strength and damage tolerance assessment}

\subsection{Static strength analysis}

Using the same solid model employed in the foundry process simulation, a mesh amenable to mechanical analysis is generated for the component (here with EDS-

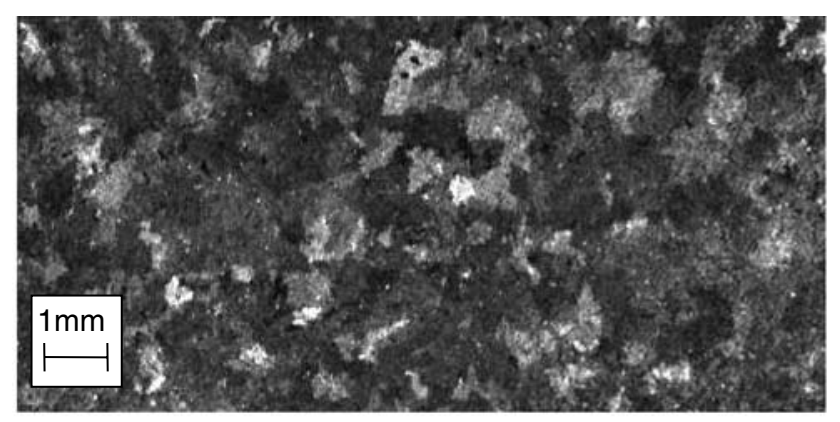

Experiment

(a) Experiment

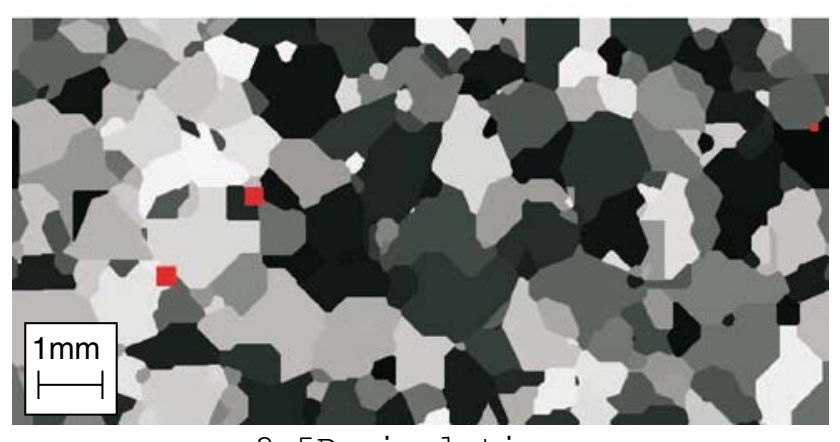

2.5D simulation

(b) $2.5 \mathrm{D}$ model

Fig. 8 Macrostructures of a experiment, b two and one-half dimensional model
PLM/I-DEAS ${ }^{\circledR}$ ). The present mesh is composed of both shell elements, for the thinner parts, and continuum elements, for the regions of interest or the bulky parts of the component. An example of such a mesh is given in Fig. 9.

The full static strength analysis of the door is repeated elsewhere in [12]. It shows that the areas in the component that show the highest stress values are the stops where the door is linked to the fuselage of the aircraft.

The casting process simulations showed that the stop regions are also prone to the formation of anomalies. This region was thus chosen as the target region to introduce initial flaws and assess the damage tolerance of the component.

Propagating arbitrary cracks in three-dimensional (3D) is a complex issue, especially if remeshing is required at each crack growth increment. Recently, a finite element technique was developed [13], which allows growth of arbitrary cracks in a fixed mesh. This extended finite element method (XFEM) was further generalized to allow the analysis of complex 3D components such as the Boeing door at hand $[12,14]$. This methodology is briefly described in Sect. 4.2.

\subsection{The super-element/XFEM and level set methodology}

\subsubsection{Introduction}

The basic approach of $[12,14]$ is summarized in Fig. 10. The complex geometrical features of the component are handled in the commercial code (I-DEAS ${ }^{\circledR}$ by EDSPLM used here) while the fracture-related issues are handled by the XFEM [13] code. The use of XFEM suppresses the need for meshing or remeshing the crack faces as the crack advances, thereby greatly alleviating the computational costs of the (possibly non-planar) 3D crack growth analysis. See [14] for a description of the interface between a $\mathrm{C}++$ research code and EDSPLM/I-DEAS ${ }^{\circledR}$ and implementation details. An advantage of XFEM is that its implementation does not require significant changes to an existing FEM code as shown in $[15,16]$, where the implementation of an open source $\mathrm{C}++\mathrm{XFEM}$ code is detailed.

\subsubsection{Crack growth methodology}

The general methodology to compute the stress intensity factors on the front of a 3D crack subject to cyclic loading and to deduce its fatigue life is outlined here:

1. Compute the displacement and strain/stress field in the structure using the XFEM approximation.

2. Compute the stress intensity factors using domain integral techniques at each point on the crack front. In this case, points on the front are taken at the intersection of the zero level set function describing 
the crack and the elements of the mesh used for the description of the mechanical and level set fields.

3. Compute the crack advance "velocity" at each of the points on the front. Here, the "velocity" at point $i$ on the front is given by the Paris law as $C\left(\Delta K_{i}\right)^{m}$. In case of mixed mode loading, $\Delta K_{i}$ is an equivalent stress intensity factor accounting for all three fracture modes.

4. Advance the crack by an amount $\Delta a_{i}=C\left(\Delta K_{i}\right)^{m} \Delta t$, where $\Delta t$ is chosen smaller than the critical time step associated with the level set update, i.e., the smallest time that it takes to the crack to cross the elements cut by the front $[12,14]$.

5. Go to (1).

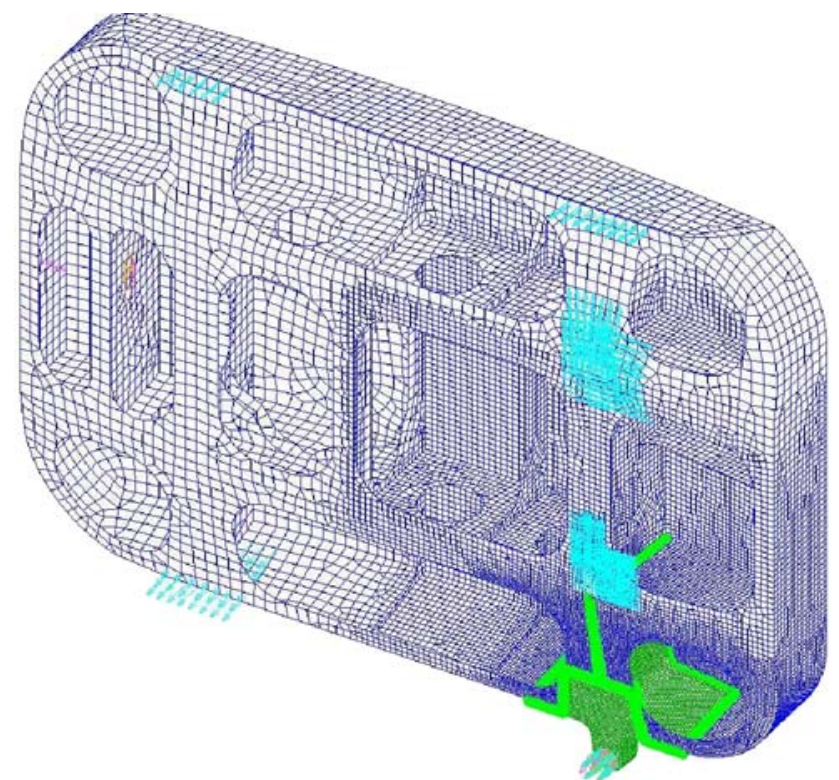

(a) Global model composed of shell and continuum elements

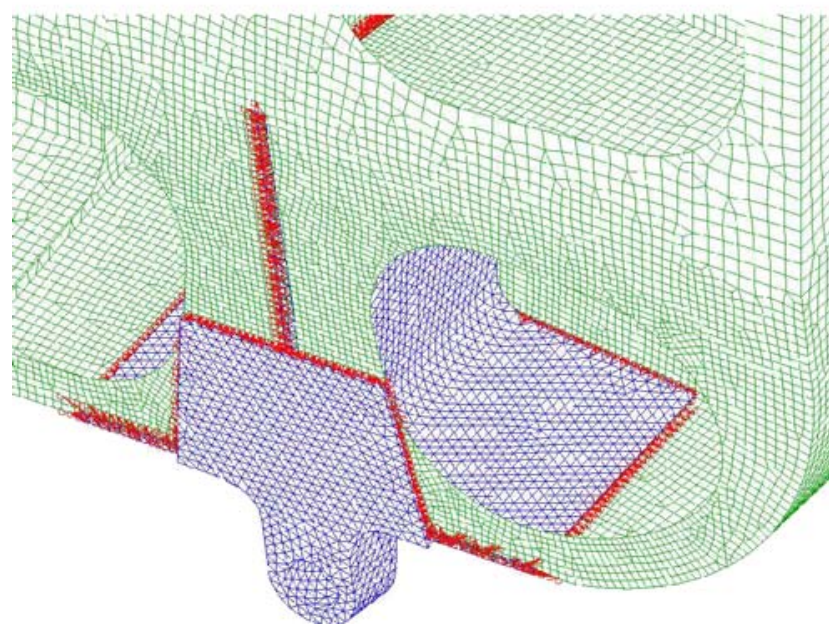

(b) Detail of the mesh of the stop region. Continuum and shell elements are linked using Multiple Point Constraint (MPC) elements.

Fig. 9 Example mesh of the Boeing 757 EE Access door used for stress and Damage Tolerance analyses
At each node on the front, the crack growth direction is computed using the maximum hoop stress criterion. Once the direction of propagation in the plane normal to the crack front passing through the current point is known, crack growth increments are required in order to know how much the crack should be advanced.

\subsubsection{Damage tolerance analysis}

The goal of the DTA is, based on the data available from the casting modeling and NDE simulations, to estimate the initial and recurring inspection intervals for the component.

Due to the versatility of the superelement procedure described above, several crack growth scenarios may easily be analyzed, often with a single mesh. A detailed DTA of the Boeing door is presented in [12, 17], and only two cases were chosen for demonstration purposes in this paper.

A possible crack growth scenario is that of an edge crack located at the peak stress location in one of the stops. Figure 11 shows the stop and crack geometry as well as the crack growth curve (crack length vs. number of cycles). This crack growth scenario yields a life of about 80 million cycles for the component. Another crack growth scenario is that of a circular crack embedded in a stop, as shown in Fig. 12.

Figure 13 shows the evolution in time of the crack. Note that the bottom half part of the crack is under

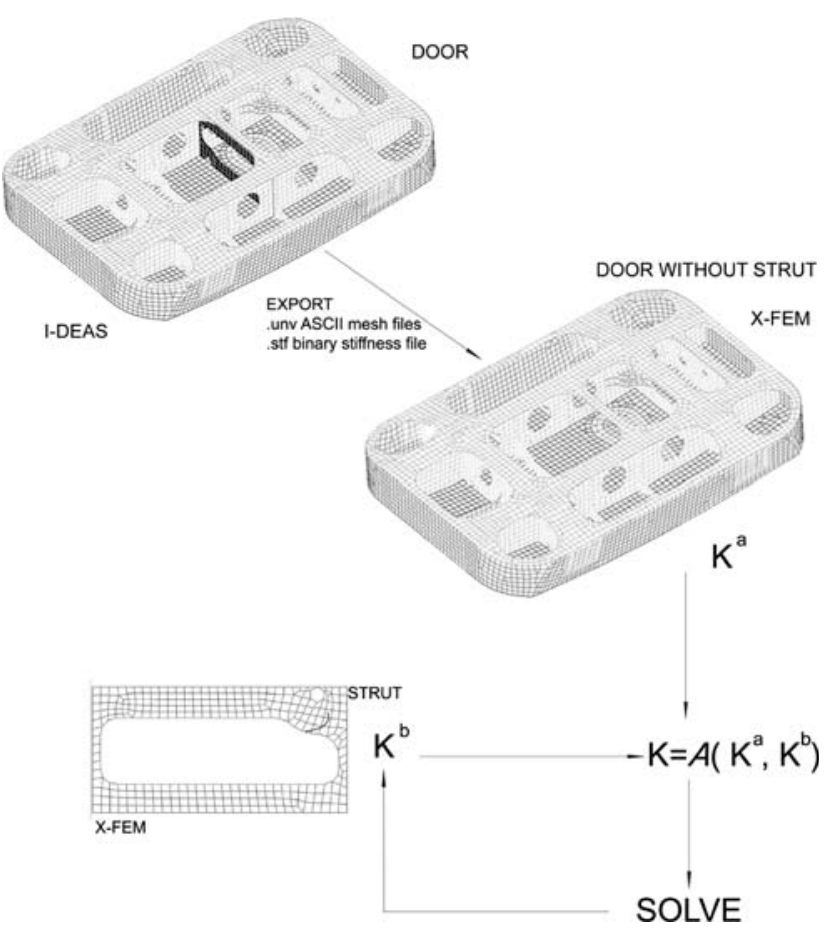

Fig. 10 Super-element/XFEM methodology, conceptual representation 
tension (opening mode) and therefore tends to open and propagate, while the top-half part of the crack is located in a compressive region and propagates a much smaller amount. The method resolves this phenomenon naturally, without any additional modification. Contact between the crack faces is not accounted for here. It is clear from the results presented in this section that the edge crack case is the most critical and is the one used to compute the recurring and initial inspection intervals (see $[12,17]$ for more details).

In practice, the initial inspection is based on the crack growth analysis using the worst case rogue flaw that would exist at manufacture and would not be detected during the manufacturing inspection. For the component under consideration, this flaw is taken as a 0.1 in corner flaw. To insure that the part be inspected several times before the flaw becomes critical, a safety factor of 2-4 is applied to the number of cycles needed to grow the flaw to its critical length. The analyses carried out here show that the current design of the door is redundant since analyses indicate that it would take multiple economic lifetimes to grow the cracks to failure, even with the visibly obvious condition of a broken stop. The broken stop scenario and the quarter edge crack configuration form the most critical situation, for which the initial inspection interval should be performed after about 750,000 cycles. The economic life of a Boeing 757 is about 50,000 flights, corresponding to a 20 year life. The inspection interval retained would be the end of one service life $(50,000$ flights).

The recurring inspection is determined by assuming the existence of the largest flaw to escape a fleet inspection. For the Boeing door, such a flaw is taken to be a 0.25 in corner flaw. The recurring inspection interval is defined by the number of cycles necessary to grow this flaw to critical divided by a safety factor to ensure multiple inspections. Here, a failed stop configuration is used as a conservative loading to lower the risk. The critical flaw size is then based on the residual strength load for the part, the proof pressure (1.5 times the operating pressure) is used to define the residual strength load and hence, the critical flaw size. The XFEM simulations provide a corresponding life of about one million cycles, which leads to a 250,000 cycle in-service inspection interval, still well above the service life of a Boeing 757. In practice however, the recurring
Fig. 11 Corner edge crack in a stop of the door, configuration and growth

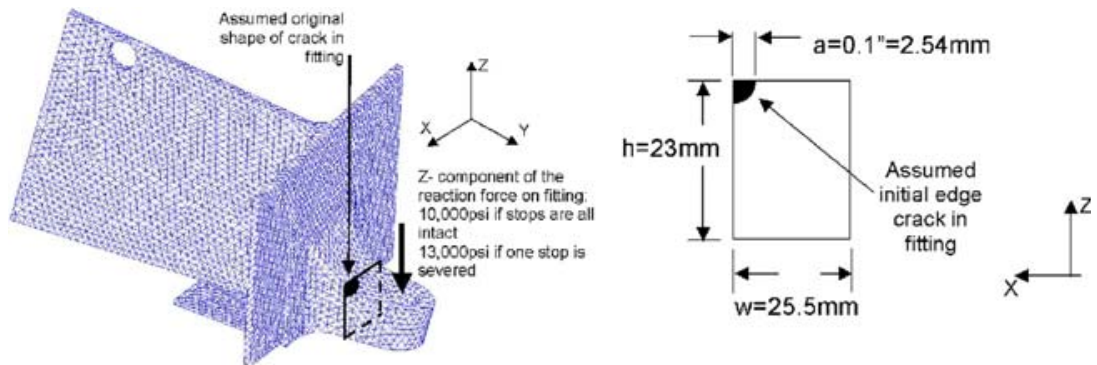

(a) Initial cracked conguration

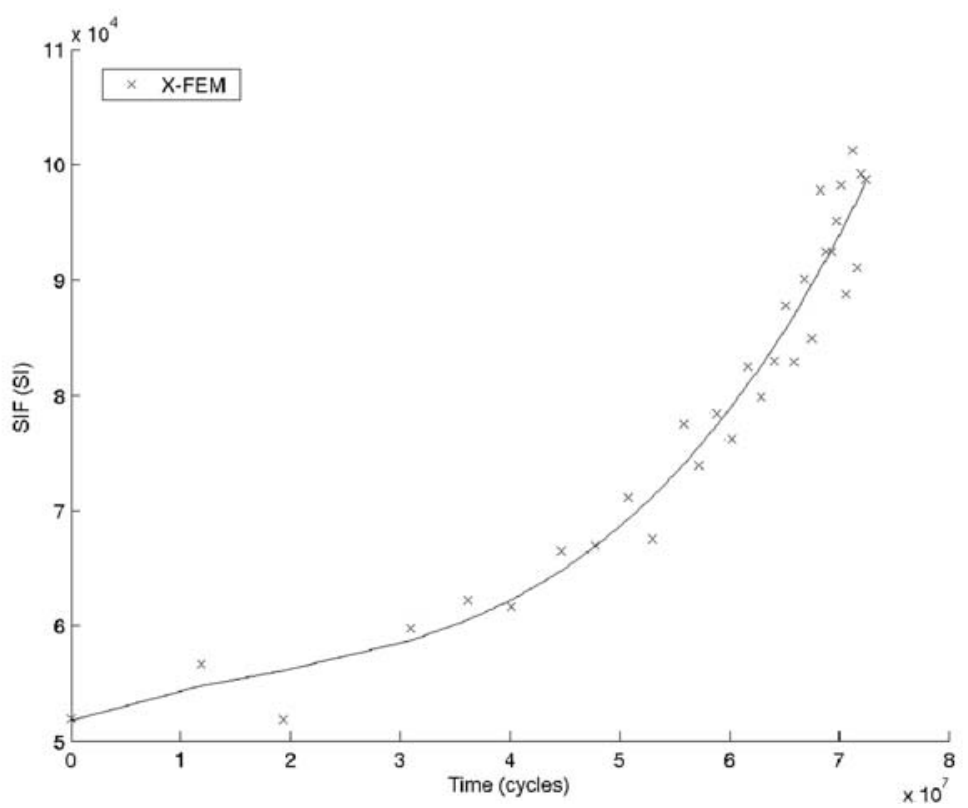

(b) Crack length $(\mathrm{mm})$ versus number of cycles 


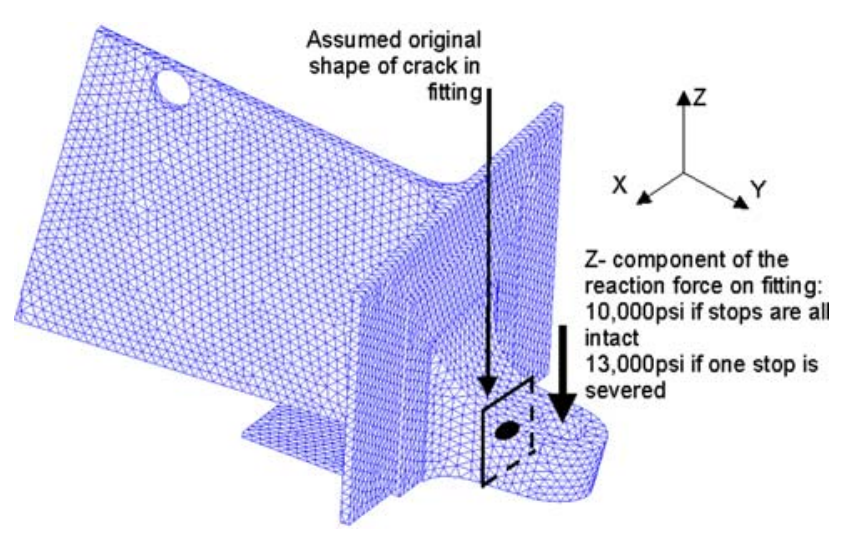

Fig. 12 Center circular crack in a stop of the door

inspections would be conducted at a scheduled maintenance check (every 7,000 flights).

This section showed how the XFEM can be used, in conjunction with commercial software, to help assess the damage tolerance of complex structures based on the results given by the casting process simulation, with a minimal amount of remeshing.

The conclusion of the damage tolerance analysis is that the door is presently over-designed so that weight could be safely removed by, for instance, decreasing the stop section area.

\subsection{NDE modeling}

A final conceptual element of the integrated design approach to the B757 EE Access door casting geometry involves simulation of the NDE process specified for the door. For the purpose of this paper, we assume that the NDE process is radiographic. The corresponding simulation helps to determine if coverage is adequate and, where appropriate, to assess the probability of detection of anomalies predicted by the foundry simulation and identified as critical by the stress and damage tolerance analyses.

The following series of images (Figs. 14, 15) illustrates how the package known as XRSIM [7]. is used to simulate the X-ray evaluation of the Boeing $757 \mathrm{EE}$ Access door. Initially, the CAD model data of the specific door geometry given in Fig. 2 is assessed for the purposes of coverage, that is how many views are required to adequately inspect the door. Note that this is the same CAD model data that were used to undertake the foundry process simulation.

The role of a thickness map is to illustrate the efficacy of the particular inspection regime. The coverage map can be seen as the negative image of the thickness map, showing the X-ray coverage of the casting. Generating multiple coverage maps for various X-ray beam orientations allows for the construction of a probability of detection map which, after being calibrated against the human eye sensitivity, indicates, at any point in the casting, the probability of detecting an anomaly of a given size [7].

The simulated radiographs shown in Fig. 15 were obtained using the XRSIM package, from the CAD data available for the Boeing $757 \mathrm{EE}$ Access door shown in Fig. 2. The anomalies that are indicated on the figures were manually chosen as an input to the simulation in order to demonstrate functionality of the software and are not representative of defects determined to be present in the subject casting.

\section{Conclusions}

This paper presented a novel integration design approach for premium quality castings in which casting process simulations, static strength analysis and DTA simulations are coupled with nondestructive evaluation simulations. The methodology is applied to the geometry, loading, casting processes and inspection methods used in the production of the Boeing 757 EE Access door, a monolithic casting of D357 alloy produced by sand casting techniques.

The work presented in this paper shows how the combination of three state-of-the-art simulation tools may help make sound design decisions at the early stage of the casting design process. The casting modeling simulation provides the designer with the locations of critical regions of the castings, and an approximate size of the largest anomalies. The damage tolerance analysis uses this anomaly as an initial flaw size to carry out fatigue crack propagation simulations and obtain the fatigue life of the component. In turn, the NDE simulation quantifies the detectability of the initial anomaly, and that of the crack in which it may grow. With this information, the designer can make sound decisions and decide rationally whether the part should be accepted or rejected in its current design. A few iterations of the
Fig. 13 Crack evolution from an initial large pore in a stop
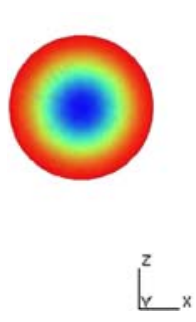
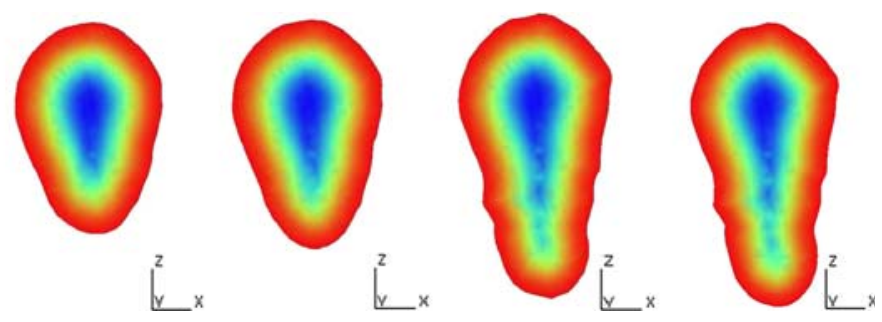


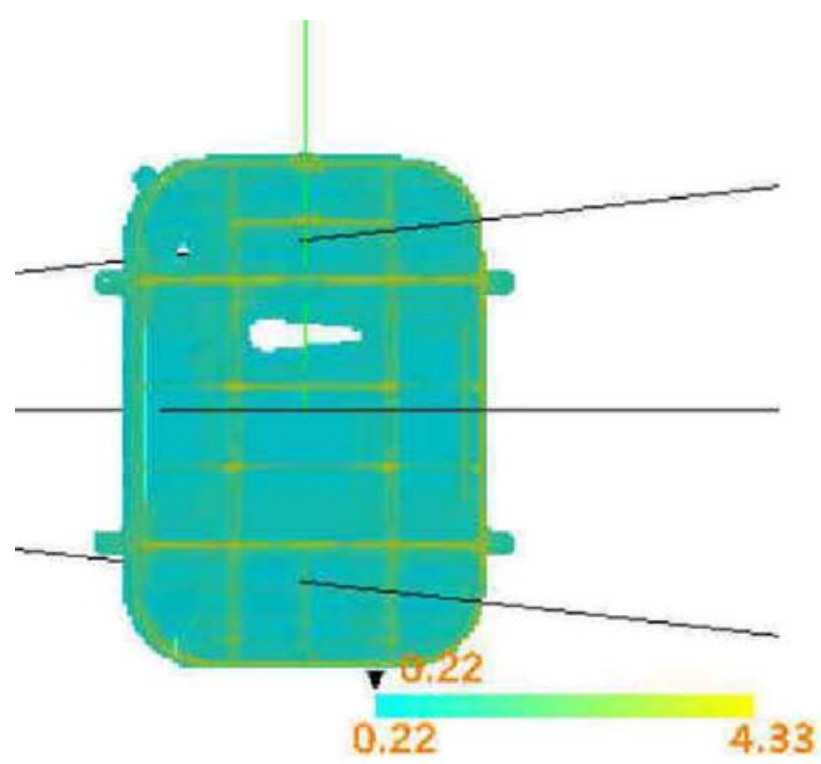

(a) Thickness map
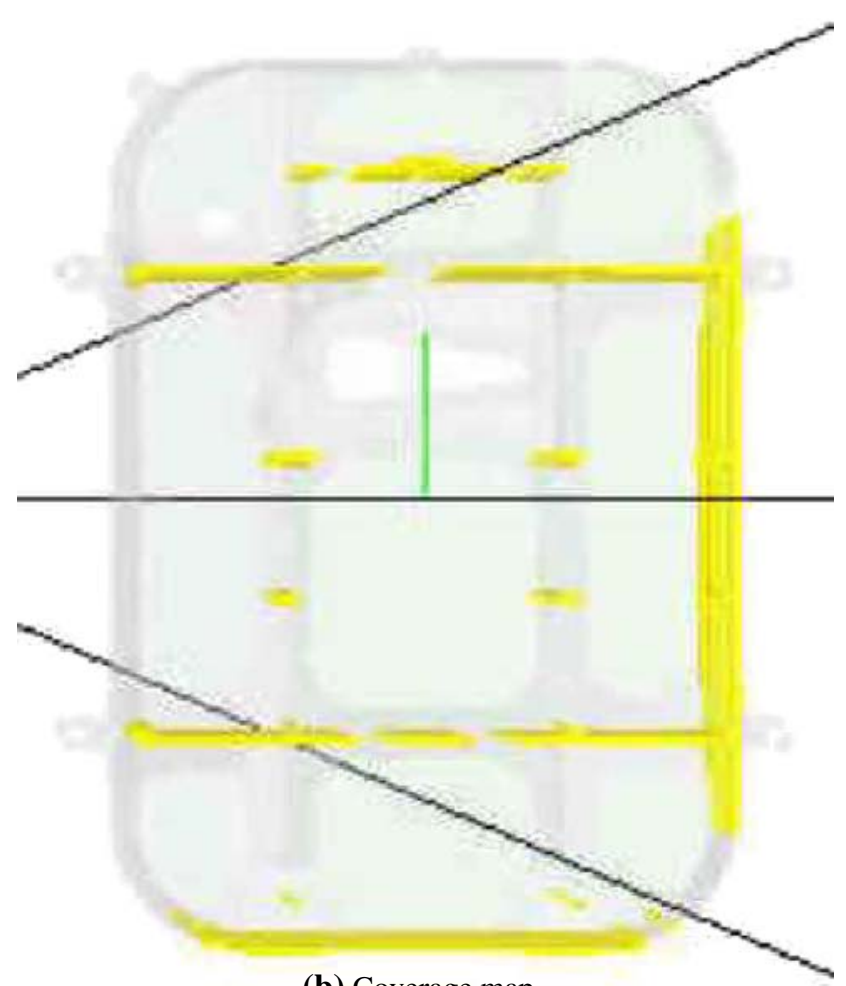

(b) Coverage map

Fig. 14 Thickness (a) and coverage (b) maps for a particular radiographic inspection configuration of the Boeing 757 EE access door

process should allow for good visibility into the component's behavior and an improvement of its design.

The numerical methods reported in each stage of the simulation analysis are based on state-of-the-art techniques. The XFEM allows modeling crack growth without remeshing and with relatively coarse meshes. However, experience in fracture mechanics and numerical analysis is still required to generate optimized meshes and

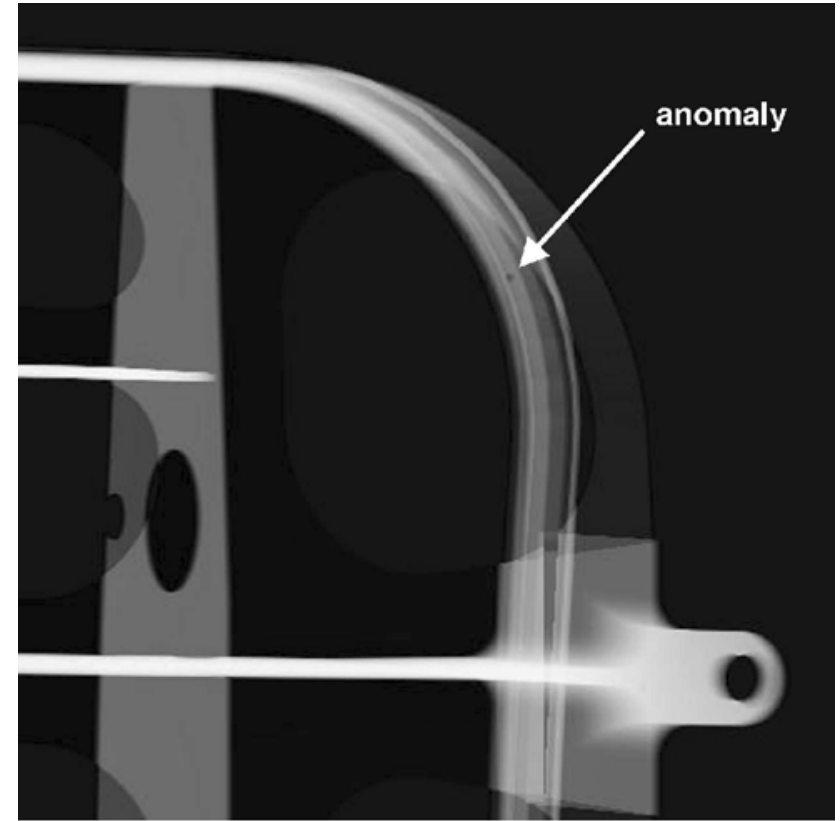

(a) Pore in the gasket area

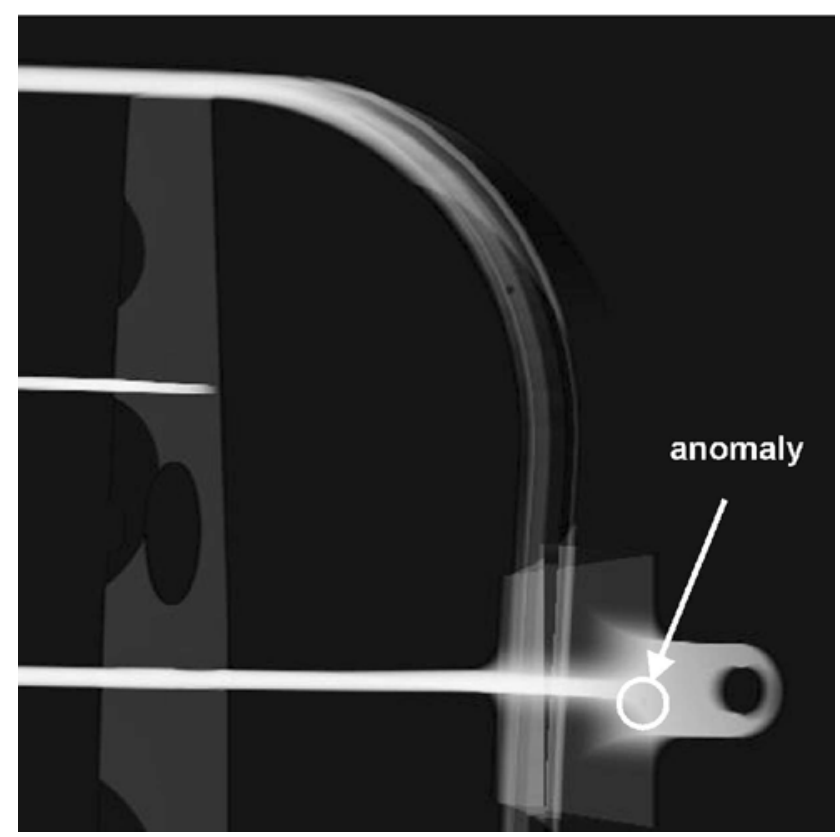

(b) Pore in the stop area

Fig. 15 Simulated radiographs of the Boeing 757 EE access door: $\mathbf{a}$ pore in the gasket area and $\mathbf{b}$ pore in the stop area. size of simulated pore is on the order of $1 \mathrm{~mm}$ in diameter. Pore is centered in the through thickness of the stop

obtain reliable results. Casting simulations lead to problem sizes of the order of hundreds of million control volumes, which, today, require recourse to parallel computing strategies. A certain level of understanding of the solidification process and of the casting microstructure evaluation is often required to fully leverage the results. The NDE simulations yield simulated X-ray images, thickness maps, coverage maps and other information 
that can be used to address probability of detection. The mastery of the techniques outlined above requires significant skills and the understanding of various fields of mechanics, numerical analysis and computer science.

Note that the analyses shown in this paper heavily rely on CAD data. Several formats exist to describe numerically geometrical entities (IGES, STEP, UNV, to name a few). It is often difficult to ascertain compatibility between the different formats, which makes communicating CAD data often long and tedious. Level set and Partition of Unity technologies in association with digital imaging is being further developed in various research groups [1822]. Research endeavours seem to tend toward the use of digital images to produce pixel (2D) or voxel (3D) data, which in turns permits an implicit definition of the part of interest. This voxel information is then used to initialize an implicit function which takes the value one inside the solid and zero outside. By gradually refining the pixel (voxel) grid, an accurate representation of the part geometry can be achieved. This permits work to be done without CAD representation of the part. The mesh can then be a regular mesh of hexahedra (quadrilaterals in 2D): the interior being defined by a non-zero value of the implicit function, the exterior by a zero value and some elements being partly inside and outside of the solid. With this technique and the rapidly growing power of computers (Moore's law predicts its doubling every 18 months), it will become increasingly easy to solve multi-million degree of freedom problems, fully continuum models of complex components would be seamlessly constructed. In addition, Partition of Unity technology is being further developed to include the effect of complex internal microstructures [18], which would allow the use of those models for advanced materials such as composite materials.

By increasing awareness and understanding of the properties and structural behavior of castings, during and after fabrication, the integrated design approach described may help quantify the aforementioned historical uncertainty and aid in making a better determination of the need for CFs.

Acknowledgments The authors gratefully acknowledge the support of the FAA through FAA contract DTFA03-98-F-IA025 Design and Quality Assurance of Premium Quality Aerospace Castings. Special thanks is extended to Xiaogong Lee, John Backuckas and Terry Khaled of the FAA for their support and availability throughout the project. The authors especially appreciated the direction, encouragement and support of the late Richard Topp of the Boeing Company.

\section{References}

1. Rice RC, Jackson JL, Bakuckas J, Thompson S (2002) Mil-m8856 metallic materials properties development standards (mmpds) handbook. Scientific Report, U.S. Department of Transportation, Federal Aviation Administration, Office of Aviation Research, January 1, 2002-December 31, 2002, used to be known as MIL-HDBK-5
2. Scoville T (1998) The emergence of aluminum castings in commercial airframes. ASM International, Materials Solutions, Rosemont, pp 117-125

3. Kearney AL (1973) The use of aluminum castings in aerospace structures. In: Transactions of the American foundrymen's society, pp 383-387

4. Allison D (1999) The application of titanium castings for commercial structure in airplanes. In: Proceedings of IMECE, American Society of Mechanical Engineers, NYC

5. Chong D (1993) Use of titanium castings without a casting factor. In: Transactions of the American foundrymen's society, pp 261-266

6. Conley JG, Moran B, Gray J (1998) A new paradigm for the design of safety critical castings. J Mater Manuf SAE Trans 106:25-38 used to be known as MIL-HDBK-5

7. Gray J (2000) Recent developments of an X-ray $N D E$ simulation tool, keynote. In: Preben N, Hansen Peter R, Sahm, James G, Conley (eds) Modeling of casting, welding and advanced solidification processes IX, ninth international conference on modeling of casting, welding and advanced solidification processes, August 20-25, 2000

8. Conley JG, Huang J, Asada J, Akiba K (2000) Modeling the effects of cooling rate, hydrogen content, grain refiner and modifier on microporosity formation in al a356 alloys. Mater Sci Eng A 285(1-2):49-55

9. Huang J, Conley JG (1998) Simulation of microporosity formation in modified and unmodified a356 alloy castings. Metall Mater Trans 29B(12):1249-1260

10. Huang J, Conley JG (1998) Computer simulation of pore size and shape for equiaxed aluminum alloy castings. Trans Am Foundry Soc 29(6):1249-1260

11. Conley JG, Moran B, Bordas S (2003) Integrated design approach of aerospace castings. Design and Quality Assurance of Premium Quality Aerospace Castings, FAA contract DTFA0398-F-IA025. Northwestern University, Center for Quality Engineering and Failure Prevention, McCormick School of Engineering and Applied Science

12. Bordas SPA (2003) Extended finite element method and level set methods with applications to the growth of cracks and biofilms. PhD thesis, Northwestern University

13. Belytschko T, Black T (1999) Elastic crack growth in finite elements with minimal remeshing. Int $\mathbf{J}$ Numer Meth Eng 45:601-620

14. Bordas S, Moran B (2006) Extended finite element and level set method for damage tolerance assessment of complex structures. Eng Fract Mech 73(9):1176-1201

15. Bordas S, Nguyen VP, Dunant C, Nguyen-Dang H, Guidoum A (2006) An object-oriented extended finite element library. Int $\mathrm{J}$ Numer Meth Eng (in press)

16. Stéphane P, Bordas S, Legay A (2005). Enriched finite element short course: class notes. In: The extended finite element method, a new approach to numerical analysis in mechanics: course notes. Organized by S. Bordas and A. Legay through the EPFL school of continuing education, Lausanne, Switzerland, December 7-9, 2005

17. Moran B, Bordas S, Conley JG (2003) Damage tolerance assessment of complex aerospace structures. Design and Quality Assurance of Premium Quality Aerospace Castings, FAA contract DTFA03-98-F-IA025, 2003. Northwestern University, Center for Quality Engineering and Failure Prevention, McCormick School of Engineering and Applied Science

18. Bordas A, Ronald H, Hoppe W, Petrova SI (2006) Mechanical failure in microstructural heterogeneous materials. In: Lecture notes in computer science (LNCS) post-proceedings. Proceedings of the sixth international conference on numerical methods and applications - NM\&A'06, Borovets, Bulgaria, August 2426, 2006 (in press)

19. Belytschko T, Parimi C, Moës N, Usui S, Sukumar N (2003) Structured extended finite element methods of solids defined by implicit surfaces. Int J Numer Meth Eng 56:609-635 
20. Moës N, Cloirec M, Cartraud P, Remacle J-F (2003) A computational approach to handle complex microstructure geometries. Comput Meth Appl Mech Eng 192:3163-3177

21. Hollister SJ, Kikuchi N (1994) Homogeneization theory and digital imaging: a basis for studying the mechanics and design principles of bone tissue. Biotech Bioeng 94(43):586-596
22. Wentorf R, Collar R, Shepard MS, Fish J (1999) Automated modeling for complex woven microstructures. Comput Meth Appl Mech Eng 172(1-4):493-506 